\title{
Interaction between gemcitabine and mitomycin-C in vitro
}

Received: 19 November 1998 / Accepted: 21 June 1999

\begin{abstract}
Both gemcitabine (2',2'-difluorodeoxycytidine; $\mathrm{dFdCyd}$ ) and mitomycin-C (MMC) are active against several solid malignancies. $\mathrm{dFdCyd}$ is an attractive agent for use in combination with drugs which damage DNA and with radiation therapy because of its ability to inhibit DNA replication and repair as well as its radiosensitizing effect. We hypothesized that the repair of MMC adducts in DNA might be inhibited by dFdCyd leading to a synergistic effect. To test this possibility, we studied the effect of combining dFdCyd and MMC in HT29 human colon carcinoma cells in vitro. The cells were exposed to a variety of drug concentration ratios and schedules, then assessed for clonogenic survival. $\mathrm{D}_{50}$ values (drug concentration at which clonogenicity is inhibited by $50 \%$ ) were calculated, and the interactive effects of the two drugs were evaluated using median effect analysis. In this approach, if the calculated combination index $(\mathrm{CI})$ is $<1,1$, or $>1$, it indicates synergism, additivity, or antagonism, respectively (Chou and Talalay 1984). We found that marked synergy (CI of $0.5-0.7)$ was produced by concurrent exposure to mitomycin and gemcitabine. In contrast, sequential treatment led only to additivity. These findings suggest that, when combined in an appropriate schedule, the chemosensitizing effect of gemcitabine may be beneficial in the treatment of malignancies which are sensitive to MMC.
\end{abstract}

Key words Gemcitabine $(\mathrm{dFdCyd}) \cdot$ Mitomycin-C (MMC) $\cdot$ Colorectal cancer

T.T. Aung - W.D. Ensminger

Division of Hematology/Oncology,

Department of Internal Medicine,

University of Michigan,

Ann Arbor, Mich., USA

M.A. Davis · T.S. Lawrence $(\bowtie)$

Department of Radiation Oncology,

B2C490 University of Michigan Medical Center,

1500 E. Medical Center Drive,

Ann Arbor, MI 48109, USA

e-mail: tsl@umich.edu

Tel.: + 1-734-647-9955; Fax: +1-734-763-7371

\section{Introduction}

Gemcitabine (2',2'-difluorodeoxycytidine; $\mathrm{dFdCyd})$, an antimetabolite, is a fluorinated deoxycytidine analog with preclinical activity in experimental tumor models as well as an impressive clinical activity in solid tumors when used singly or in combination $[2,4,7,15,17,21,24]$. Mitomycin-C (MMC), a bifunctional alkylating agent, has shown therapeutic activity against a variety of cancers especially in combination with other agents $[8,34]$. To date, little is known about the potential effect of the combination $\mathrm{dFdCyd}$ and MMC. Only one in vitro study has been reported using this combination of drugs on a Lewis lung (LL) non-small-cell lung cancer cell line [22].

A crucial step in dFdCyd cytotoxicity is its phosphorylation by deoxycytidine kinase [14]. The resulting phosphorylated metabolites exhibit multiple cellular effects: (a) they prevent DNA synthesis by inhibiting DNA polymerases and by competing with deoxycytidine triphosphate [16, 25], (b) they deplete nucleotide pools by inhibiting ribonucleotide reductase [3, 29], and (c) they decrease the accuracy of DNA replication and repair by incorporation into DNA [16, 27]. In addition to its cytotoxic activity, $\mathrm{dFdCyd}$ is a potent radiation sensitizer for a variety of human tumor cell lines [28]. Thus, dFdCyd which is cell cycle specific, is an attractive agent for use in combination with DNA-damaging drugs and radiation therapy. On the other hand, MMC, a bioreductive agent which is not cell cycle specific in its actions, is unique among the alkylating agents in that it can be metabolized to an active species which crosslinks complementary DNA strands, thereby inhibiting DNA synthesis [9]. After bioreduction and chemical reduction of MMC has occurred, guanine is the preferred site of alkylation of DNA [33], but this crosslinking is slowly repairable [20].

Since both $\mathrm{dFdCyd}$ and MMC have been reported to have enhanced activity in combination with other DNAdamaging agents $[6,11,13,26,32]$, we postulated that $\mathrm{dFdCyd}$ and MMC together may have complementary activity, leading to a selective synergism against the 
targeted tumor cells. To test this hypothesis, we studied the effect of combining dFdCyd and MMC in HT29 human colon carcinoma cells in vitro. Our goal was to establish whether the combination of these two drugs has a synergistic effect and if any schedule dependency exists. In assessing schedule, we wished to test both the dependence on the order of drug treatments and the effect of delaying the determination of clonogenic survival for $24 \mathrm{~h}$ after $\mathrm{dFdCyd}$ treatment. This latter part of the design derived from our finding that dFdCyd cytotoxicity and radiosensitization were increased under these conditions [19]. We found that concurrent exposure to dFdCyd and MMC produced the most synergistic effect in vitro. This finding supports the development of clinical trials using concurrent $\mathrm{dFdCyd}$ and $\mathrm{MMC}$ for the treatment of solid malignancies.

\section{Materials and methods}

\section{Cell culture/drugs}

Human colon adenocarcinoma cells (HT29) from the American Type Culture Collection (Manassas, Va.) were cultured in RPMI1640 medium supplemented with glutamine $(10 \mathrm{mM})$, penicillin $(100 \mathrm{IU} / \mathrm{ml})$, and $10 \%$ bovine calf serum. Cells were maintained at $37{ }^{\circ} \mathrm{C}$ in a humidified atmosphere of $7 \% \mathrm{CO}_{2}$ and $93 \%$ air as a monolayer under conditions allowing for exponential growth. Under these conditions the plating efficiency of HT29 cells was 70$90 \%$, and the doubling time was approximately $21 \mathrm{~h}$. dFdCyd (Eli Lilly Research Laboratories, Indianapolis, Ind.) was dissolved in phosphate-buffered saline (PBS), and all exposures were for $2 \mathrm{~h}$ at $37^{\circ} \mathrm{C}$. MMC (Bristol Laboratories, Princeton, N.J.) for clinical use $(40 \mathrm{mg} / 80 \mathrm{ml})$ was dissolved in PBS, and all incubations were carried out for $1 \mathrm{~h}$ at $37^{\circ} \mathrm{C}$. Incubations were carried out with the drugs diluted from frozen stocks which were made fresh every 3 months. In experiments where the dishes were to be processed $24 \mathrm{~h}$ after the end of the drug exposure, the drug-containing medium was removed, the dishes washed with PBS, and fresh medium added.

Fig. 1A,B Effect of dFdCyd and MMC on the clonogenic survival of HT29 colon cancer cells. A Cells were exposed to dFdCyd for $2 \mathrm{~h}$, the drug was washed from the medium, and the cells were then processed for clonogenic survival immediately $(\square)$ or $24 \mathrm{~h}$ later ( as described in Material and methods. B Cells were exposed to MMC for $1 \mathrm{~h}$ and then processed for clonogenic survival as described in Materials and methods. The values are the mean \pm standard error of at least three experiments

\section{Cell survival assay}

Cell survival was assessed following exposure to $\mathrm{dFdCyd}$ and MMC using a standard clonogenic assay as described previously [18]. Briefly, at the time of processing, the medium was removed, the dishes were washed with PBS, and the cells were removed from the plate using trypsin. After the cells had been removed from the dishes, they were counted and diluted into dishes at numbers that were anticipated to produce between 20 and 200 colonies per plate. Appropriate controls of each drug alone were performed in all cases. All experiments were repeated at least three times, with the results expressed as means \pm standard error of at least three experiments. In order to apply the median effect analysis (see below), data were fitted using the equation:

Surviving fraction $=\mathrm{C}_{50}^{M} /\left(\mathrm{C}_{50}^{M}+\mathrm{C}^{M}\right)$

where $\mathrm{C}$ is the concentration of drug, $\mathrm{C}_{50}$ is the concentration that reduces the surviving fraction to $50 \%$, and $M$ is the slope of the sigmoid curve. The fit was performed using a logarithmic transformation to linearize the equation, as described by Chou and Talalay [5]. We also calculated the linear area under the cell survival curve in a calculation analogous to that used to estimate radiation sensitivity [10]. A smaller area suggests greater sensitivity to the drug condition.

Analysis of cell survival assay

In a first set of experiments, a low, noncytotoxic fixed concentration of dFdCyd and variable doses of MMC were used. The sensitivity of the cells to drug was expressed by calculating the area under the cell survival curve, so that a smaller area indicates greater sensitivity. Potential enhancement of MMC cytotoxicity by $\mathrm{dFdCyd}$ was expressed by calculating the ratio of the area under the curve under control conditions divided by the area under dFdCyd-exposed conditions, so that a number greater than 1 would indicate increased sensitivity. In a second set of experiments, combinations of $\mathrm{dFdCyd}$ and MMC in various cytotoxic ranges of concentration were analyzed according to the median effect principle as described by Chou and Talalay [5]. The interaction of the two chemotherapeutic agents was then quantified by calculating the combination index (CI). The CI can be calculated by assuming a mutually nonexclusive interaction.

Within the methodology of the median effect calculation, there is a need to determine whether an interaction is "mutually exclusive" or "non-mutually exclusive". Our calculation of the CI as non-mutually exclusive presupposes no assumptions about the mechanism of killing by either drug. This approach adds a term to the CI $\left(\mathrm{D}_{1} \mathrm{D}_{2} / \mathrm{Dx}_{1} \mathrm{Dx}_{2}\right)$ compared to the mutually exclusive assumption of interaction and of isobologram analysis [5]. Since higher CIs are interpreted as indicating less enhancement (or even antagonism when the index is greater than 1), we feel that we have made a conservative estimate of the enhancement by using the nonmutually exclusive assumption [5] by the equation:

$C I=\left(D_{1} / D x_{1}\right)+\left(D_{2} / D x_{2}\right)+\left(D_{1} D_{2} / D_{1} D_{2}\right)$
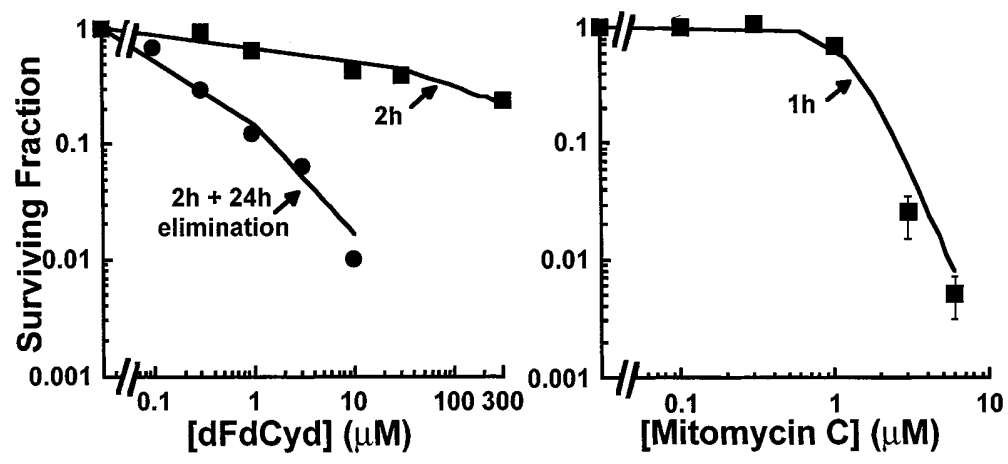
where $\mathrm{Dx}_{1}$ and $\mathrm{Dx}_{2}$ are the concentrations of drugs 1 and 2, when used alone which produce a surviving fraction of $\mathrm{x}$, and $\mathrm{D}_{1}$ and $\mathrm{D}_{2}$ are the individual concentrations of drugs 1 and 2 in the fixed ratio which produces a surviving fraction of $\mathrm{x}$. In this combination model, an antagonistic relationship between the agents is implied when the CI value is greater than 1 , while a CI value of 1 indicates an additive effect. A CI value less than 1 denotes synergism.

Statistical analysis

Data are expressed as the means \pm standard error of at least three experiments. Means were compared using Student's $t$-test.

\section{Results}

Our experimental design was derived from our experiments using $\mathrm{dFdCyd}$ with radiation. In these studies we found that $\mathrm{dFdCyd}$ could radiosensitize under noncytotoxic conditions. Therefore, we began by determining the cytotoxicity of dFdCyd and of MMC against HT29 cells. For dFdCyd, cells were treated over a 2-h period. The drug was then washed from the medium, and the cells were assessed for clonogenic survival immediately or $24 \mathrm{~h}$ later. This time period was chosen because the effects of dFdCyd on dNTP pool depletion occur over the first $30 \mathrm{~min}$ and have plateaued by $2 \mathrm{~h}$ [14]. For MMC, clonogenicity was determined immediately after a 1-h drug exposure (Fig. 1). We found that the $\mathrm{D}_{50}$ (drug concentration at which clonogenicity is inhibited by $50 \%$ ) of cells assessed $24 \mathrm{~h}$ after exposure to $\mathrm{dFdCyd}$ was significantly lower than the $\mathrm{D}_{50}$ of cells assessed immediately after drug treatment (Table 1). This difference in surviving fraction between cells which are assessed immediately versus those that are assessed $24 \mathrm{~h}$ after a brief drug exposure is consistent with our previous observations and suggests that immediate trypsinization has a protective effect [19]. We could then ascertain whether, as was the case for radiation [19], noncytotoxic concentrations of $\mathrm{dFdCyd}$ could sensitize cells to MMC. We then determined the clonogenic survival of HT29 cells exposed to noncytotoxic dFdCyd $(0.1 \mathrm{~m} M$ for $2 \mathrm{~h}$ ) with MMC either in the 2nd hour (concurrent) or $24 \mathrm{~h}$ after $\mathrm{dFdCyd}$ treatment (sequential) (Table 1, Fig. 2). These experiments revealed that unlike radiation, noncytotoxic concentrations of dFdCyd did not enhance MMC cytotoxicity.

Table 1 Cytotoxicities of $\mathrm{dFdCyd}$ and MMC. Cells were treated with various concentrations of $\mathrm{dFdCyd}$, or with various concentrations of $\mathrm{MMC}$ with or without $0.1 \mu M \mathrm{dFdCyd}$ according to the indicated schedule and assessed for clonogenic survival. The results were fitted to the equation: surviving fraction $=\mathrm{D}_{50}^{M} /$

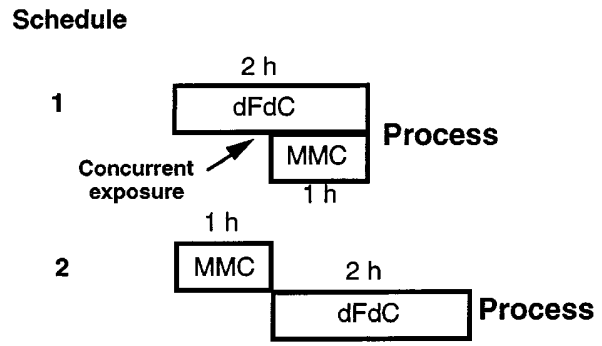

3

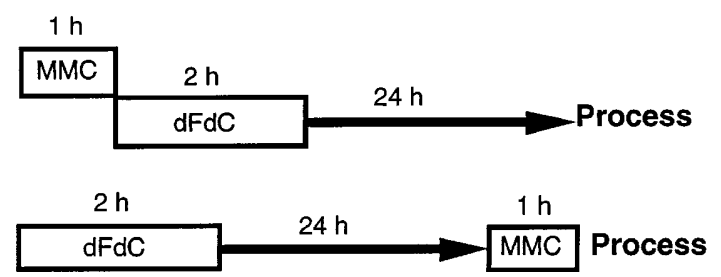

Fig. 2 Experimental design of the study

Since it was possible that synergy required both agents to be used under cytotoxic conditions, we examined different cytotoxic dose ratios of the two drugs using median effect analysis. First, it was necessary to determine whether the relationship between drug concentration and surviving fraction for both the individual drugs and the drugs in combination could be fitted by a sigmoid curve. We found that this was the case (Fig. 3; data not shown for schedules 2, 3, and 4). We were then in a position to determine the dependence of drug interaction on sequence (schedules 1 and 2; Fig. 2). A comparison of these conditions would reveal whether synergy required MMC or dFdCyd to be administered first. Each of the above schedules was investigated using concentration ratios of $\mathrm{dFdCyd}$ to MMC of 1:1, 10:1, and $1: 10$. We found that concurrent exposure (schedule 1) produced CIs consistently below 1 for all concentration ratios. This finding suggests a strong synergistic interaction between the two drugs. On the other hand, MMC followed by dFdCyd (schedule 2) did not show synergy. In fact, the CIs for schedule 2 were suggestive of additivity or even antagonism (CIs greater than 1; Table 2).

When we found that the schedule of MMC followed by dFdCyd did not produce synergy (schedule 2), we assessed the same schedule after a 24-h delay to permit

$\left(D_{50}^{M}+D^{M}\right)$ where $D_{50}$ is the concentration that reduces the surviving fraction to $50 \%$, and $M$ is the slope of the sigmoid curve. The area under the cell survival curve was calculated as described in Materials and methods; a smaller area indicates greater drug sensitivity

\begin{tabular}{lllll}
\hline Drugs & Condition & $\mathrm{D}_{50}(\mathrm{~m} M)$ & $M$ & AUC \\
\hline MMC & 1 h exposure, process immediately & $1.16 \pm 0.17$ & $3.88 \pm 0.60$ & $1.31 \pm 0.16$ \\
DFdCyd (various concentrations) & 2 h exposure, process immediately & $5.84 \pm 0.99$ & $1.46 \pm 0.66$ & $5.30 \pm 0.73$ \\
DFdCyd (0.1 $\mu M)+$ MMC & MMC during 2nd hour of dFdCyd & $0.95 \pm 0.46$ & $3.24 \pm 0.82$ & $1.11 \pm 0.45$ \\
DFdCyd (various concentrations) & 2 h exposure, process 24 h later & $0.18 \pm 0.05$ & $0.94 \pm 0.11$ & $0.71 \pm 0.16$ \\
DFdCyd $(0.1 \mu M)+$ MMC & MMC, 24 h after dFdCyd & $0.80 \pm 0.02$ & $4.09 \pm 0.25$ & $0.88 \pm 0.01$ \\
\hline
\end{tabular}




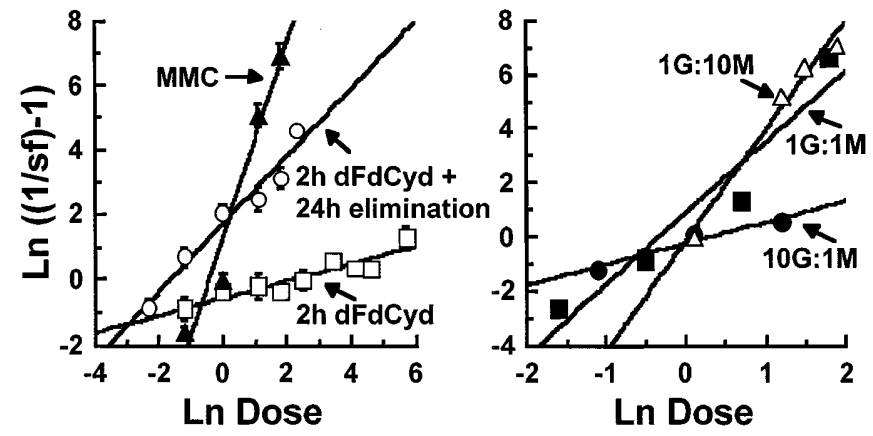

Fig. 3A,B Assessment of appropriateness of median effect analysis for $\mathrm{dFdCyd-MMC}$ interactions. A Cells were exposed to $\mathrm{dFdCyd}$ or MMC, assessed for clonogenicity, and the data were fitted to the equation $\ln (1 /$ (surviving fraction $)-1)=m \ln (\mathrm{C})-m \ln \left(\mathrm{C}_{50}\right)$, as described in Materials and methods. B Cells were exposed to fixed ratios of $\mathrm{dFdCyd}$ and $\mathrm{MMC}$ under the conditions described in schedule 1. For example, with a fixed ratio of $1: 1$, a total concentration of $6 \mu M$ indicates $3 \mu M \mathrm{dFdCyd}$ and $3 \mu M$ MMC. The values are the mean \pm standard error of at least three experiments. Correlation coefficients for all lines are greater than 0.85

any effects of dFdCyd on the repair of MMC lesions to develop (schedule 3). Evaluation of the CIs resulting from treatment with MMC followed by $\mathrm{dFdCyd}$ with delayed processing (schedule 3) produced a complex pattern suggesting, in general, additivity, although it is possible that synergy existed at a ratio of 1:1 (Table 2).

In addition, we wished to assess whether synergy occurred in cells treated with MMC $24 \mathrm{~h}$ after dFdCyd treatment (schedule 4). These conditions produce potent radiosensitization of HT29 cells [19]. Unlike the radiosensitization seen previously, we found that this sequence (schedule 4) overall suggested additivity with CIs near 1 (Table 2).

\section{Discussion}

In this study, we wished to determine whether combinations of $\mathrm{dFdCyd}$ and MMC would be synergistic. A concurrent schedule of $\mathrm{dFdCyd}$ and MMC combination produced the largest synergistic effect under these conditions as based on the criteria set by Chou and Talalay [5]. In contrast, exposure to MMC following dFdCyd treatment had either an additive or an antagonistic effect. These findings are consistent overall with those of a

Table 2 Combination indices for all ratios of $\mathrm{dFdC}: \mathrm{MMC}$ at surviving fraction $(S F)$ of 0.1 and 0.03 . Cells were treated with various concentrations of $\mathrm{dFdCyd}$ and $\mathrm{MMC}$ at a fixed ratio of previous study investigating the effects of the combination of dFdCyd and MMC against a non-small-cell lung cancer line. In that study, dFdCyd and MMC were synergistic after a 4-h exposure when given at a constant molar ratio (dFdCyd:MMC 1:4), but after a 24-72-h exposure only additivity was produced.

A number of potential mechanisms could explain $\mathrm{dFdCyd-MMC}$ synergy. One possibility is that $\mathrm{dFdCyd}$ increases MMC crosslinking or decreases crosslink removal. Another possibility is that MMC increases dFdCyd-induced DNA double strand breaks, although this was not found in the study alluded to above [22]. A less likely possibility is that $\mathrm{dFdCyd}$ may affect MMC metabolism by modulating a bioreductive pathway (such as DT-diaphorase [30]). Further study will be required to determine which of these and other possibilities is the most likely.

Regardless of the mechanism for dFdCyd-MMC synergy, it is clear that dFdCyd interacts differently with radiation compared to MMC. In contrast to our findings with $\mathrm{dFdCyd}$ and $\mathrm{MMC}$, radiosensitization is produced under noncytotoxic $\mathrm{dFdCyd}$ conditions and when $\mathrm{dFdCyd}$ treatment precedes radiation by $24-48 \mathrm{~h}$. This suggests that the nature of the DNA damage is a key determinant of $\mathrm{dFdCyd}$-mediated sensitization.

$\mathrm{dFdCyd}$ has also been examined in combination with cisplatin. A synergistic interaction between the combination of dFdCyd and cisplatin in vitro as well as in vivo in animal studies and also in clinical studies has been reported [4, 23]. However, experimental studies of this in vitro combination have revealed that the synergistic effect is much more pronounced when cisplatin exposure precedes $\mathrm{dFdCyd}$ exposure, unlike our findings which showed an opposite effect.

An important issue in interpreting the results of this study concerns the method which we used to assess synergy. Although the median effect principle is a wellestablished method of assessing drug-drug interactions, other methods, such as dose-surface response [12] and isobologram analysis [31] have also been used. Each approach has its proponents, and it is not clear at this time which of these methods produces more clinically relevant results. In addition, the overall lack of cytotoxicity from $\mathrm{dFdCyd}$ alone, at the clinically achievable concentrations that we chose [1], in cells processed immediately after trypsin treatment makes it more difficult to quantify synergy. However, the finding of synergy for

10:1, 1:1 or 1:10 (dFdCyd:MMC). The combination indices were then calculated, as described in Materials and methods, at a surviving fraction of 0.1 or 0.03

\begin{tabular}{|c|c|c|c|c|c|c|}
\hline Schedule & \multicolumn{3}{|l|}{$\mathrm{SF}=0.1$} & \multicolumn{3}{|l|}{$\mathrm{SF}=0.03$} \\
\hline 2 & $2.75 \pm 1.20$ & $1.15 \pm 0.12$ & $1.72 \pm 0.31$ & $3.43 \pm 1.70$ & $1.03 \pm 0.15$ & $1.30 \pm 0.18$ \\
\hline 3 & $0.91 \pm 0.10$ & $0.60 \pm 0.04$ & $1.42 \pm 0.08$ & $0.82 \pm 0.07$ & $0.44 \pm 0.05$ & $1.08 \pm 0.08$ \\
\hline 4 & $1.32 \pm 0.23$ & $0.92 \pm 0.15$ & $1.23 \pm 0.40$ & $1.01 \pm 0.09$ & $0.61 \pm 0.13$ & $1.04 \pm 0.1$ \\
\hline
\end{tabular}


both the delayed and relatively cytotoxic schedule 3 and the immediate and relatively noncytotoxic schedule 1 suggests that $\mathrm{dFdCyd}$ chemosensitization occurs under a range of conditions.

Based on these findings, we have designed a phase I trial of combination MMC and dFdCyd for patients with advanced refractory malignancies. MMC is administered at its usual dose of $10 \mathrm{mg} / \mathrm{m}^{2}$ every 6-week cycle for two cycles, and dFdCyd is dose-escalated from a starting dose $500 \mathrm{mg} / \mathrm{m}^{2}$ given weekly for 12 weeks within two cycles of treatment. A total of six patients have been accrued, and dose-limiting toxicity has not yet been reached. Although it is too early to judge the efficacy of this approach, we hope that the antitumor cell synergy observed in vitro will translate to the clinic.

\section{References}

1. Abbruzzese JL, Grunewald R, Weeks EA, Gravel D, Adams T, Nowak B, Mineishi S, Tarassoff P, Satterlee W, Raber MN, et al (1991) A phase I clinical, plasma, and cellular pharmacology study of gemcitabine. J Clin Oncol 9: 491

2. Abratt R, Bezwoda W, Falkson G, Goedhals L, Hacking D, Rugg T (1994) Efficacy and safety profile of gemcitabine in nonsmall-cell lung cancer: A phase II study. J Clin Oncol 12: 1535

3. Baker $\mathrm{CH}$, Banzon J, Bollinger JM, Stubbe J, Samano V, Robins MJ, Lippert B, Jarvi E, Resvick R (1991) 2'-Deoxy$2^{\prime}$-methylenecytidine and 2'-deoxy-2',2'-difluorocytidine $5^{\prime}$-diphosphates: potent mechanism-based inhibitors of ribonucleotide reductase. J Med Chem 34: 1879

4. Braakhuis BJ, Dongen GA van, Vermorken JB, Snow GB (1995) Preclinical in vivo activity of $2^{\prime}, 2^{\prime}$-difluorodeoxycytidine (Gemcitabine) against human head and neck cancer. Cancer Res 51: 221

5. Chou T-C, Talalay P (1984) Quantitative analysis of doseeffective relationships: the combined effects of multiple drugs or enzyme inhibitors. Adv Enzyme Res 22: 27

6. Comis R (1979) Mitomycin C in gastric cancer. In: Carter SK, Crooke ST (eds) Mitomycin C: current status and new developments. Academic Press, New York San Francisco London, p 129

7. Crino L, Scagliotti G, Marangolo M (1997) Cisplatin-gemcitabine in advanced non-small cell lung cancer: a phase II study. J Clin Oncol 15: 297

8. Crooke S, Bradner W (1976) Mytomycin C: a review. Cancer Treat Rev 31: 121

9. Dorr R (1988) New findings in the pharmacokinetic, metabolic, and drug-resistance aspects of mitomycin-C. Semin Oncol 4: 32

10. Fertil B, Dertinger H, Courdi A, Malaise EP (1984) Mean inactivation dose: a useful concept for intercomparison of human cell survival curves. Radiat Res 99: 73

11. Godfroy T (1979) Mitomycin C in breast cancer. In: Carter SK, Crooke ST (eds) Mitomycin C: current status and new developments. Academic Press, New York San Francisco London, p 91

12. Greco WR, Park HS, Rustum YM (1990) Application of a new approach for the quantitation of drug synergism to the combination of cis-diamminedichloroplatinum and 1-beta-Darabinofuranosylcytosine. Cancer Res 50: 5318

13. Grunwald R, Akrivakis K, Mergenthaler H, Blatter J, Possinger K (1998) Phase I study of gemcitabine and epirubicin for metastatic breast cancer (abstract 241). Proceedings of the 15th Annual Meeting of the American Society of Clinical Oncology

14. Heinemann V, Hertel LW, Grindey GB, Plunkett W (1988) Comparison of the cellular pharmacokinetics and toxicity of $2^{\prime}, 2^{\prime}$-difluorodeoxycytidine and 1- $\beta$-D-arabinofuranosylcytosine. Cancer Res 48: 4024
15. Hertel LW, Boder GB, Kroin JS, Rinzel SM, Poore GA, Todd GC, Grindey GB (1990) Evaluation of the antitumor activity of gemcitabine $\left(2^{\prime}, 2^{\prime}\right.$-difluoro-2'-deoxycytidine). Cancer Res 50: 4417

16. Huang P, Chubb S, Hertel LW, Grindey GB, Plunkett W (1991) Action of 2',2'-difluorodeoxycytidine on DNA synthesis. Cancer Res 51: 6110

17. Kaye SB (1994) Gemcitabine: current status of phase I and II trials (editorial). J Clin Oncol 12: 1527

18. Lawrence TS, Davis MA, Maybaum J, Stetson PL, Ensminger WD (1990) The effect of single versus double-strand substitution on halogenated pyrimidine-induced radiosensitization and DNA strand breakage in human tumor cells. Radiat Res 123: 192

19. Lawrence TS, Chang EY, Hahn TM, Shewach DS (1997) Delayed radiosensitization of human colon carcinoma cells after a brief exposure to $2^{\prime}, 2^{\prime}$-difluoro-2'-deoxycytidine (Gemcitabine). Clin Cancer Res 3: 777

20. Matsumoto A, Vos JM, Hanawalt PC (1989) Repair analysis of mitomycin C-induced DNA crosslinking in ribosomal RNA genes in lymphoblastoid cells from Fanconi's anemia patients. Mutat Res 217: 185

21. Moore M, Andersen J, Burris H. Tarassoff P, Green M, Casper E, Portenoy R, Modiano M, Cripps C, Nelson R (1995) A randomized trial of gemcitabine (GEM) versus $5 \mathrm{FU}$ as first-line therapy in advanced pancreatic cancer (abstract 473). Proceedings of the 14th Annual Meeting of the American Society of Clinical Oncology

22. Moorsel C van, Veerman G, Bergman A, Guechev A (1997) Combination chemotherapy studies with gemcitabine. Semin Oncol 24 [suppl 7]: 17

23. Peters G, Bergman A, Ruiz van Haperen V, Veerman G, Kuipe RC, Braakhius B (1995) Interaction between cisplatin and gemcitabine in vitro and in vivo. Semin Oncol 22 [Suppl 11]: 72

24. Rothenberg ML, Moore MJ, Cripps MD, Anderson JS, Portenoy RK, Burris HA, Green MR (1996) A phase II trial of gemcitabine in patients with 5-FU-refractory pancreas cancer. Ann Oncol 14: 347

25. Ruiz van Haperen VWT, Veerman G, Vermorken JB, Peters GJ (1993) 2',2'-difluorodeoxycytidine (gemcitabine) incorporation into RNA and DNA of tumour cell lines. Biochem Pharmacol 46: 762

26. Sandler A, Ansari R, McClean J (1995) Gemcitabine plus cisplatin in non-small cell lung cancer: a phase II study. Eur J Cancer 131A: S255

27. Schy WE, Hertel LW, Kroin JS, Bloom LB, Goodman MF, Richardson FC (1993) Effect of a template-located 2',2'difluorodeoxycytidine on the kinetics and fidelity of base insertion by Klenow ( $3^{\prime}-5^{\prime}$ exonuclease-) fragment. Cancer Res 53: 4582

28. Shewach DS, Lawrence TS (1996) Gemcitabine and radiosensitization in human tumor cells. Invest New Drugs 14: 257

29. Shewach DS, Hahn TM, Chang E, Hertel LW, Lawrence TS (1994) Metabolism of $2^{\prime}, 2^{\prime}$-difluoro-2'-deoxycytidine and radiation sensitization of human colon carcinoma cells. Cancer Res 54: 3218

30. Siegel D, Gibson N, Preush P, Rose D (1990) Metabolism of mitomycin C by DT-diaphorase: role in mitomycin C-induced DNA damage and cytotoxicity in human colon carcinoma cells. Cancer Res 50: 7483

31. Steel GG, Peckham MJ (1979) Exploitable mechanisms in combined radiotherapy-chemotherapy: the concept of additivity. Int J Radiat Oncol Biol Phys 5: 85

32. Teicher B, Devita V, Hellman S, Rosenberg S, Devita VT, Hellman S, Rosenberg SA (1977) Cancer, principles and practice of oncology, 4th edn. Lippincott, Philadelphia, p 405

33. Tomasz M, Chawala A, Lipman R (1988) Mechanism of monofunctional and bifunctional alkylation of DNA by mitomycin C. Biochemistry 27: 3182

34. Verweij J (1997) Mitomycins. Cancer Chemother Biol Response Modif 16: 48 